\title{
A comparison of pneumolysin activity and concentration in vitro and in vivo in a rabbit endophthalmitis model
}

\author{
Melissa E Sanders' \\ Erin W Norcross' \\ Quincy C Moore III' \\ Chinwendu Onwubiko' \\ Lauren B King' \\ Jonathan Fratkin² \\ Mary E Marquart' \\ 'Department of Microbiology; \\ ${ }^{2}$ Department of Pathology, University \\ of Mississippi Medical Center, Jackson, \\ MS, USA
}

\begin{abstract}
The purpose of this study was to determine whether the in vitro activity and concentration of Streptococcus pneumoniae pneumolysin correlated to the pathogenesis of $S$. pneumoniae endophthalmitis. Five $S$. pneumoniae clinical endophthalmitis strains were grown in media to similar optical densities (OD), and extracellular milieu was tested for pneumolysin activity by hemolysis of rabbit red blood cells. Pneumolysin concentration was determined using a sandwich ELISA. Rabbit vitreous was injected with $10^{2}$ colony-forming units (CFU) of 1 of 2 different strains with low hemolytic activity ( $\mathrm{n}=10$ and 12 for strains 4 and 5 , respectively) or 1 of 3 different strains with high hemolytic activity ( $n=12$ per strain). Pathogenesis of endophthalmitis infection was graded by slit lamp examination (SLE) at 24 hours post-infection. Bacteria were recovered from infected vitreous and quantitated. The SLE scores of eyes infected with strains having high hemolytic activity were significantly higher than the scores of those infected with strains having low hemolytic activity $(\mathrm{P}<0.05)$. Pneumolysin concentration in vitro, however, did not correlate with hemolysis or severity of endophthalmitis. Bacterial concentrations from the vitreous infected with 4 of the strains were not significantly different $(\mathrm{P}>0.05)$. These data suggest that pneumolysin hemolytic activity in vitro directly correlates to the pathogenesis of $S$. pneumoniae endophthalmitis. The protein concentration of pneumolysin, however, is not a reliable indicator of pneumolysin activity.

Keywords: Streptococcus pneumoniae, endophthalmitis, pneumolysin, pneumococcal
\end{abstract}

\section{Introduction}

Staphylococcus species account for the majority of cases of bacterial endophthalmitis (Callegan et al 2007), however, infections due to Streptococcus pneumoniae (pneumococcus) lead to vision loss more rapidly than those with most other bacteria (Miller et al 2004). S. pneumoniae has been reported as a significant cause of endophthalmitis following ocular surgery (Mao et al 1992; Nouri et al 2001; Soriano et al 2006). A virulence factor of $S$. pneumoniae, pneumolysin (PLY), has been shown to be important in the pathogenesis of this disease ( $\mathrm{Ng}$ et al 1997, 2002), as well as several other diseases, including pneumonia (Rubins et al 1995; Witzenrath 2006; Garcia-Suarez Mdel et al 2007), bacteremia (Yuste et al 2005; Quin et al 2007), otitis media (Comis et al 1993; Beurg et al 2005), meningitis (Hirst et al 2008), and keratitis (Johnson et al 1990; Marquart et al 2007; Green et al 2008). A PLYdeficient strain of $S$. pneumoniae causes decreased keratitis (Johnson et al 1990), and the complement activation domain of PLY appears to be partially responsible for the clinical symptoms (Johnson et al 1995). In pneumococcal endophthalmitis, PLY has been reported to be important in the early stages of the disease and causes inflammation and retinal necrosis ( $\mathrm{Ng}$ et al 1997, 2002).
Correspondence: Mary E Marquart Department of Microbiology, $2500 \mathrm{~N}$ State Street, University of Mississippi Medical Center, Jackson, MS 39216, USA $\mathrm{Tel}+\mathrm{I} 6018156934$

$\mathrm{Fax}+\mathrm{I} 6019841708$

Emailmmarquart@microbio.umsmed.edu 
PLY is a $53 \mathrm{kDa}$ toxin that is produced intracellularly and is commonly thought to be released upon bacterial cell lysis, however, the precise mechanism of PLY release is not known. This toxin is usually located in the extracellular milieu of stationary and death phase cultures of S. pneumoniae, and does not possess any consensus secretion signal sequences. PLY belongs to the family of cholesterol-dependent cytolysins, of which Clostridium perfringens perfringolysin O (Kirkham et al 2006) and Listeria monocytogenes listeriolysin O (Mengaud et al 1987) are members. PLY and members of this toxin family bind to cholesterol in host cell membranes and form pores, often resulting in lysis of the host cell. Subcytolytic concentrations of PLY have been shown to activate host cell complement (Paton et al 1984), and this complement activation can exacerbate pneumococcal infections due to inflammation. For example, corneal inflammation and PMN infiltration are symptoms of $S$. pneumoniae keratitis, and deletion of the complement activation domain of PLY decreases the ability of S. pneumoniae to cause the inflammation observed in keratitis (Johnson et al 1995). Separate domains of the PLY molecule have been linked to its cytolytic and complement-activating functions (Jounblat et al 2003).

In this study, we quantitated PLY activity and protein concentration in 5 S. pneumoniae strains isolated from cases of endophthalmitis. These in vitro quantitations were compared to the severity of endophthalmitis caused by each corresponding strain in vivo in rabbits.

\section{Methods}

\section{Bacterial strains and growth conditions}

Clinical endophthalmitis strains of S. pneumoniae (E391, E206, E323, E461, and E353) were kindly provided by Regis Kowalski at the Charles T Campbell Eye Microbiology Lab (Pittsburg, PA, USA). For simplification, we designated strain E391 as strain 1, E206 as strain 2, E323 as strain 3, E461 as strain 4, and E353 as strain 5. Bacteria were grown overnight on 5\% sheep blood agar (BA). Todd-Hewitt Broth with $0.5 \%$ yeast extract (THY) was inoculated with one colony and incubated at $37{ }^{\circ} \mathrm{C}$ in $5 \% \mathrm{CO}_{2}$ overnight. The overnight culture was inoculated into fresh THY at a 1:100 dilution. The bacteria were grown to an optical density (OD) at $\mathrm{A}_{600}$ that corresponded to $10^{8}$ colony-forming units $(\mathrm{CFU})$ per $\mathrm{ml}$ for infection or grown overnight $\left(\mathrm{A}_{600} \approx 0.9\right)$ for evaluation of extracellular pneumolysin. Cells from the overnight culture were separated from extracellular milieu by centrifugation. Pneumolysin was quantitated from extracellular material.

\section{Hemolysis assay}

Extracellular samples were mixed with a 5\% solution of rabbit red blood cells (RBCs) in PBS and incubated in triplicate at $37{ }^{\circ} \mathrm{C}$ for 30 minutes in 96 -well round-bottom plates. The plates were centrifuged at $3200 \mathrm{rpm}$ for 5 minutes to pellet nonlysed RBCs. The supernatants were measured for hemoglobin content by spectrophotometry at $\mathrm{A}_{450}$. Saponin $(0.5 \%)$ was used as the positive control that represented $100 \%$ hemolysis. Percent hemolysis was determined by averaging the $\mathrm{A}_{450}$ values of each sample and converting the values to a percent based on the positive control.

\section{Sandwich ELISA}

The method used was previously described by Cima-Cabal (2001). In short, a 96-well ELISA plate was coated with mouse anti-PLY antibody (Affinity BioReagents, Golden, CO, USA) and incubated overnight. The subsequent steps were blocking of nonspecific proteins, application of extracellular bacterial extracts, application of polyclonal rabbit PLY-specific antiserum (Green et al 2008), application of anti-rabbit IgG alkaline phosphatase conjugate (Sigma-Aldrich, St. Louis, MO, USA), and color development. The color intensity in each well was measured spectrophotometrically at $\mathrm{A}_{405}$. Background was subtracted from each well, and protein concentrations were determined by comparison to a standard curve consisting of known concentrations of purified recombinant PLY prepared as previously described (Thornton and McDaniel 2005).

\section{Infection}

The use of animals in this research complied with the guidelines of, and was approved by, the Institutional Animal Care and Use Committee of the University of Mississippi Medical Center. New Zealand white rabbits (Harlan Sprague Dawley, Inc, Oakwood Research Facility, Inc., Oxford, MI, USA) were anesthetized by an intramuscular injection of a mixture of xylazine $(100 \mathrm{mg} / \mathrm{mL}$; Butler Company, Columbus, $\mathrm{OH}, \mathrm{USA})$ and ketamine hydrochloride $(100 \mathrm{mg} / \mathrm{mL}$; Butler Company). Proparacaine hydrochloride ( $0.5 \%$; Bausch and Lomb, Rochester, NY, USA) was topically applied to each eye before injection. Bacterial cultures were diluted such that each vitreous humor was infected with $10^{2} \mathrm{CFU}$ in a volume of $10 \mu 1$. The bacteria were inoculated into the vitreous humor of each rabbit eye with a 30 -gauge needle.

\section{Slit lamp examination (SLE)}

Eight parameters were used for determining the severity of endophthalmitis: injection, chemosis, corneal inflammation, 
anterior chamber cell, anterior chamber flare, red reflex, vitreal clarity, and retinal clarity (Callegan et al 1999). Each parameter was given a grade from 0 (no pathogenesis) to 4 (maximal pathogenesis), resulting in a total score with a theoretical maximum of 32 . Each eye was scored by 2 examiners blind to the experiment, and the two scores were averaged.

\section{CFU recovery}

Rabbits were euthanized by an overdose of intravenous sodium pentobarbital (Sigma-Aldrich) at 24 or 48 hours post infection (PI). Vitreous was removed from each eye, serially diluted, and cultured in triplicate on BA. The plates were incubated at $37^{\circ} \mathrm{C}$ in $5 \% \mathrm{CO}_{2}$ overnight and colonies were counted to determine log CFU recovered.

\section{Statistics}

Data were analyzed using the Statistical Analysis System (SAS) program for computers (SAS Inc., Cary, NC, USA). Clinical SLE scores were analyzed using a nonparametric one-way analysis of variance. Bacterial CFU at $24 \mathrm{~h}$ and $48 \mathrm{~h}$
PI, PLY activity, and PLY quantification were analyzed using the general linear models procedure of least squares means. $P<0.05$ was considered significant.

\section{Histopathology}

Whole eyes were removed and histological sectioning was performed by Excalibur Pathology, Inc. (Moore, OK, USA). Sections were stained with hematoxylin and eosin.

\section{Results \\ Hemolysis assays}

Hemolysis assays were performed to characterize each strain's PLY activity. PLY activity is based on the toxin's ability to lyse RBCs. Strains were considered to have low PLY activity if hemolysis was $<50 \%$ and high PLY activity if hemolysis was $>80 \%$ relative to a $100 \%$ lysis control. Strains 1,2 , and 3 were designated high PLY activity strains with $94.5 \%, 100 \%$, and 100\% hemolysis respectively (Figure 1A). Strains 4 and 5 were designated low PLY activity strains with $12.1 \%$ and $49.6 \%$ hemolysis, respectively.
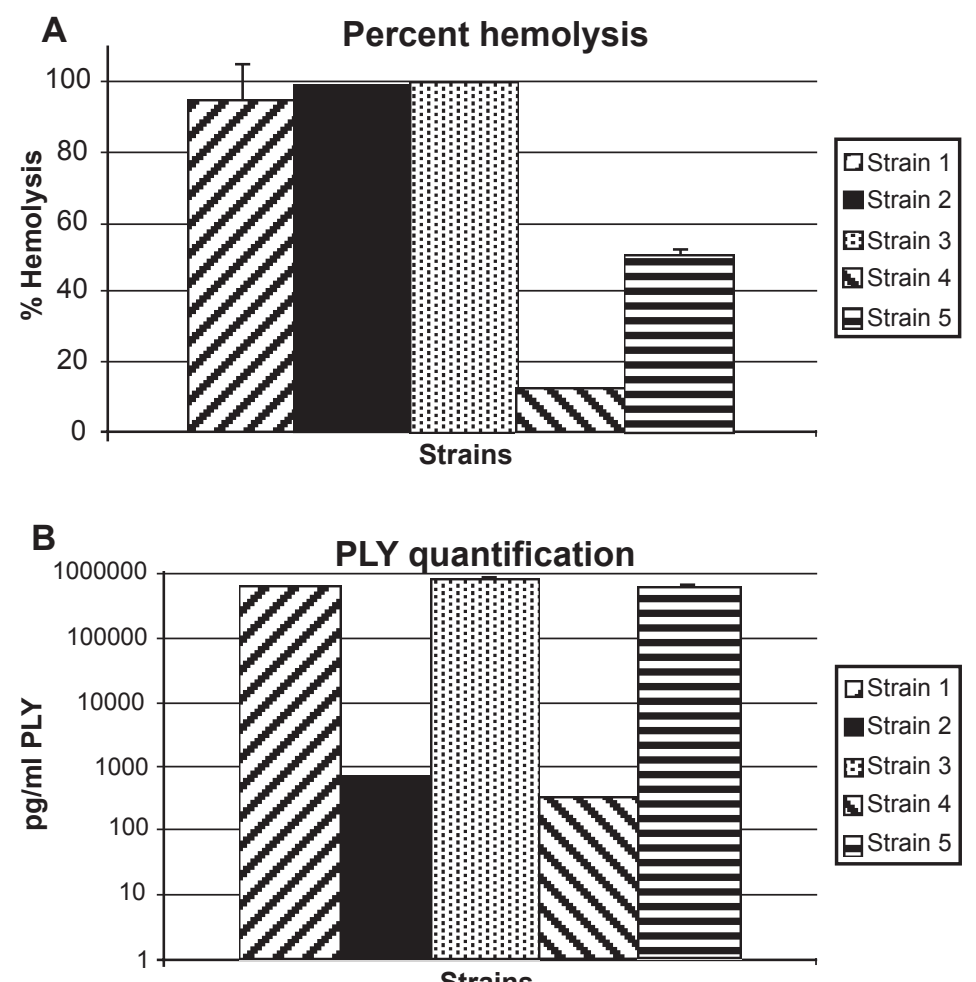

Figure I (A) Percent hemolysis ( $\mathrm{n}=3$ per strain) for study strains. Percent was determined by comparison to a saponin I00\% control. None of the high hemolytic strains $(I, 2,3)$ were significantly different from each other $(P>0.05)$. All of the high hemolytic strains were significantly higher than the low hemolytic strains $(4$ and 5$)(P<0.05)$. Strain 4 was significantly lower than strain $5(\mathrm{P}<0.000 \mathrm{I})$ Error bars denote standard deviation. (B) Concentration of PLY for study strains as determined by ELISA. Error bars denote standard deviation. Strain 3 had significantly more PLY than all other strains $(P<0.05)$. Strains I and 5 had significantly more PLY than strains 2 and 4 (P $<0.05)$. Strains $I$ and 5 and strains 2 and 4 are not significantly different $(P>0.05)$. 


\section{Sandwich ELISA}

The sandwich ELISA was used to quantify the amount of PLY released by the bacteria in overnight cultures (Figure 1B). When compared to hemolytic activity, the amount of PLY released did not correlate to the ability to lyse RBCs (Figure 1).

\section{Rabbit endophthalmitis model}

Strains with low $(<50 \%)$ and high $(>80 \%)$ extracellular hemolysis relative to a $100 \%$ lysis control were compared in a rabbit endophthalmitis model. Rabbit vitreous was injected with 1 of 2 different strains of low hemolytic activity ( $n=10$ and 12 for strains 4 and 5, respectively) or 1 of 3 different strains of high hemolytic activity $(n=12$ per strain). Strains 1, 2, and 3 had high hemolytic activity in vitro and average SLE scores \pm standard errors of the means of $23.13 \pm 2.21,17.76 \pm 2.16$, and $16.59 \pm 1.89$, respectively, at 24 hours PI. Strains 4 and 5 had low hemolytic activity and average SLE scores of $9.91 \pm 2.09$ and $10.54 \pm 2.07$, respectively, at 24 hours PI. The SLE scores of eyes infected with strains of high hemolytic activity were significantly higher than the SLE scores of eyes infected with strains of low hemolytic activity $(\mathrm{P}<0.05$; Figure 2$)$.

Figure 3 shows representative eyes from low hemolysis strains and high hemolysis strains. The high hemolysis strains caused a much more severe pathology. At both 24 and 48 hours PI, more inflammatory cells can be observed (as anterior chamber cell and flare as determined by SLE) in the eyes infected with the high activity strains as compared to the eyes infected with the low activity strains. The disease severity caused by strains 1 and 2 was so severe that the experiment

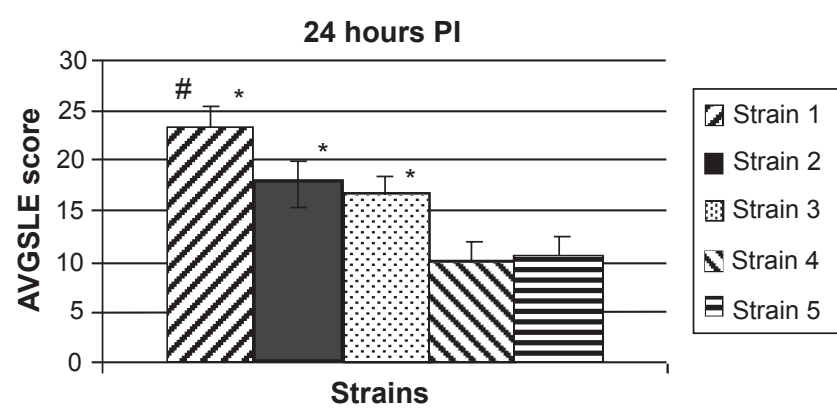

Figure 2 Average SLE scores at 24 hours PI. All strains producing high hemolytic activity in vitro (strains I, 2, and 3) caused significantly higher SLE scores in vivo than the low activity strains (strains 4 and $5 ; \mathrm{P}<0.05$ ). There was no significant difference in SLE scores between the two low activity strains $(P=0.8342)$. There were no significant differences in SLE scores between the three high activity strains $(P>0.05)$, except for strain I compared to strain $3(P=0.0352)$. Error bars denote standard errors of the means.

Notes: *significant compared to low hemolytic strains $(\mathrm{P}<0.05)$ ' " significant compared to strain $3(P=0.0352)$.

Abbrevations: $\mathrm{PI}$, post infection; SLE, slit lamp examination. was terminated after 24 hours PI, and those rabbits were euthanized. Infections were allowed to progress in the eyes infected with the remaining 3 strains until 48 hours PI.

\section{CFU recovery}

There was no significant difference in bacterial concentration from vitreous infected with strains 2, 3, 4, and 5 at 24 hours PI (P > 0.05; Table 1). Significantly higher log CFU were recovered from vitreous infected with strain 1 when compared to all other strains $(\mathrm{P}<0.05)$. There was no significant difference in bacterial concentration from vitreous infected with strains 3 and 5 at 48 hours PI $(P=0.4194)$. However, there was a significant difference in bacterial concentration from vitreous infected with strains 3 and 4 as well as strains 4 and $5(\mathrm{P}=0.0047$ and $\mathrm{P}=0.0242$, respectively).

\section{Histopathology}

Inflammation observed in the eyes infected with the high PLY activity strains was more pronounced and destructive than in the eyes infected with the low PLY activity strains. Also, inflammation of the vitreous, retina, and choroid was observed in the eyes infected with the high activity PLY producing strains. The retina had become detached from the optic disk, and had become invaginated. Inflammation was observed in the subretinal space in eyes infected with the high activity PLY producing strains. Less severe intravitreal inflammation was observed in the eyes infected with the low activity PLY producing strains. The retinas were intact, and there was little to no inflammation observed in the subretinal

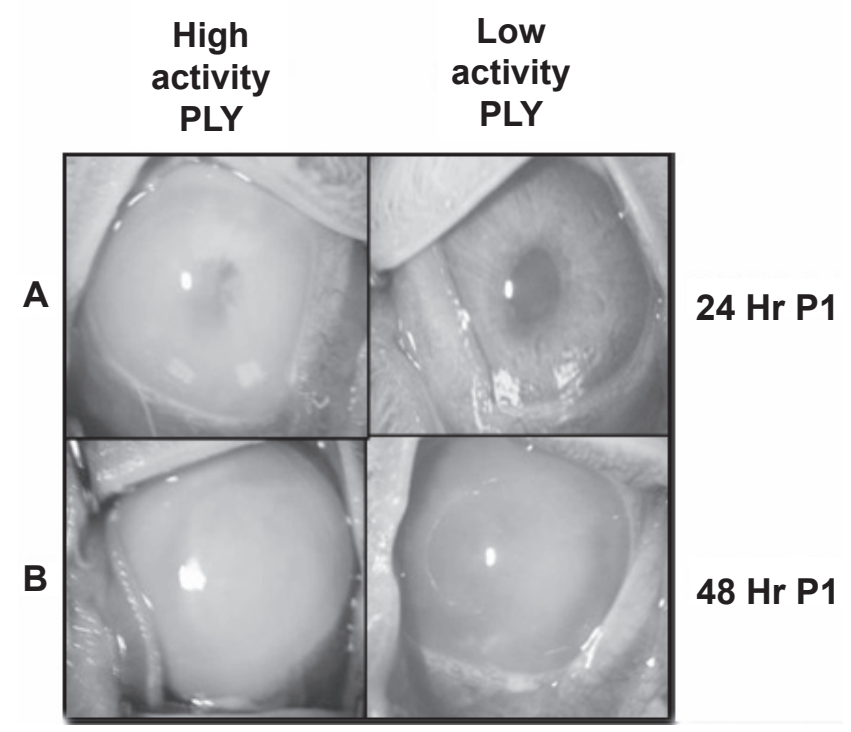

Figure 3 Pictures of representative eyes at 24 hours PI (A) and 48 hours PI (B). Strains 3 (left column) and 5 (right column) are shown. $N=12$ for all strains except strain $4(n=10)$.

Abbreviations: PI, post infection; PLY, pneumolysin. 
Table I $\log _{10}$ CFU recovered from the vitreous

\begin{tabular}{|c|c|c|}
\hline & 24 hours $\mathrm{PI}^{\mathrm{a}}$ & 48 hours $\mathrm{Pl}^{\mathrm{b}}$ \\
\hline Strain I & $9.0011 \pm 0.1204$ & N/D \\
\hline Strain 2 & $7.0479 \pm 0.3772$ & N/D \\
\hline Strain 3 & $7.6364 \pm 0.2226$ & $8.8540 \pm 0.3493$ \\
\hline Strain 4 & $7.9383 \pm 0.3844$ & $7.1227 \pm 0.4180$ \\
\hline Strain 5 & $7.9133 \pm 0.2522$ & $8.2429 \pm 0.1903$ \\
\hline
\end{tabular}

Notes: ${ }^{a} n=12$ per strain at 24 hours PI (except strain $4 ; n=10$ ). Strain I was significantly higher than all other strains at 24 hours PI $(P<0.05)$. P $>0.05$ among all other strains $(2,3,4,5)$ at 24 hours PI; 'S Strains 3 and $5 n=11$; strain $4 n=9$ at 48 hours PI. Log CFU recovered from the vitreous of eyes infected with strain 4 was significantly lower $(\mathrm{P}<0.05)$ when compared to strains 3 and 548 hours PI. Strains 3 and 5 showed no significant difference at 48 hours $\mathrm{Pl}(\mathrm{P}=0.4 \mathrm{I}$ (94). Results presented as log CFU \pm standard error of the mean

Abbreviations: CFU, colony-forming units; N/D, not determined, PI, post infection.

space in eyes infected with the low activity PLY producing strains (Figure 4).

Strains with high hemolytic activity caused destruction of the inner and outer layers of the retina and inflammatory infiltrate behind the retina was observed (Figure 4B). A dense, pre-retinal (vitreal) infiltrate of polymorphonuclear (PMN) inflammatory cells was also observed. Strains with low hemolytic activity (Figure 4C) caused only a moderate infiltration of PMN cells in the pre-retinal space. Few inflammatory cells were observed in the sub-retinal space, and the retina remained intact.

\section{Discussion}

This study sought to find correlation between the hemolytic activity of PLY from clinical endophthalmitis strains with the severity of endophthalmitis caused by those strains in a rabbit model. Though PLY is an intracellular toxin, hemolysis caused by the extracellular milieu from the overnight cultures correlated with severity of infection in vivo. This correlation is probably due to bacterial cell death in the overnight culture. It is commonly believed that upon cell death (or lysis), PLY is released. Since there is a higher amount of dead bacterial cells in an overnight culture, more PLY should be present in the milieu. Rabbit vitreous infected with $S$. pneumoniae strains producing high hemolytic activity in vitro showed more severe pathology than those infected with strains producing low hemolytic activity. The protein quantity of PLY from each of the strains, however, did not correlate with hemolytic activity. The amount of PLY also did not relate to the pathology in vivo. Strain 2, for example, had high hemolytic activity but produced relatively less PLY than strain 5, which had low hemolytic activity. These results suggest at least two scenarios. PLY activity per PLY concentration could vary from strain to strain, possibly due to mutation at the gene level or incomplete post-translational modification of the protein that would render the protein to activity ratio higher in some strains compared to others. A second possibility is that hemolytic activity is not necessarily fully attributable to PLY. Although hemolytic activity is a widely accepted indicator of PLY function, one study reported that a serotype 2 strain of $S$. pneumoniae deficient in the gene for PLY retained its hemolytic activity (Canvin et al 1997).

The hemolytic activity of each strain correlated to the severity of pathogenesis in the rabbit endophthalmitis model for all strains at 24 hours PI. For example, strains 1, 2, and 3 had high hemolytic activity and caused more severe endophthalmitis than strains 4 and 5 (Figures 1, 2, and 3). The differences observed between the strains in endophthalmitis severity were not due to differences in bacterial growth for strains 2, 3, 4 and 5 as determined by quantitation of bacterial concentration from the vitreous (Table 1). The significantly higher bacterial concentration from the vitreous of eyes infected with strain 1 could account for the increased pathogenesis observed in vivo for this strain compared to the other strains. Perhaps other factors lend to the ability of this strain to replicate in the eye.

Histology of the rabbit eyes supports the SLE observations. A larger amount of PMNs were observed in the vitreous of the rabbit eyes infected intravitreously with the strains producing high activity PLY as compared to the low activity strains. Furthermore, the retinas in the eyes infected with the high activity PLY producing strains showed more necrosis than the retinas of the eyes infected with the low PLY producing strains (Figure 4).

Only two other studies have been reported that have investigated the virulence mechanisms of $S$. pneumoniae endophthalmitis. PLY was injected into the vitreous of Lewis rats and caused rapid inflammation and tissue damage, as well as retinal necrosis and detachment ( $\mathrm{Ng}$ et al 1997). In addition, 


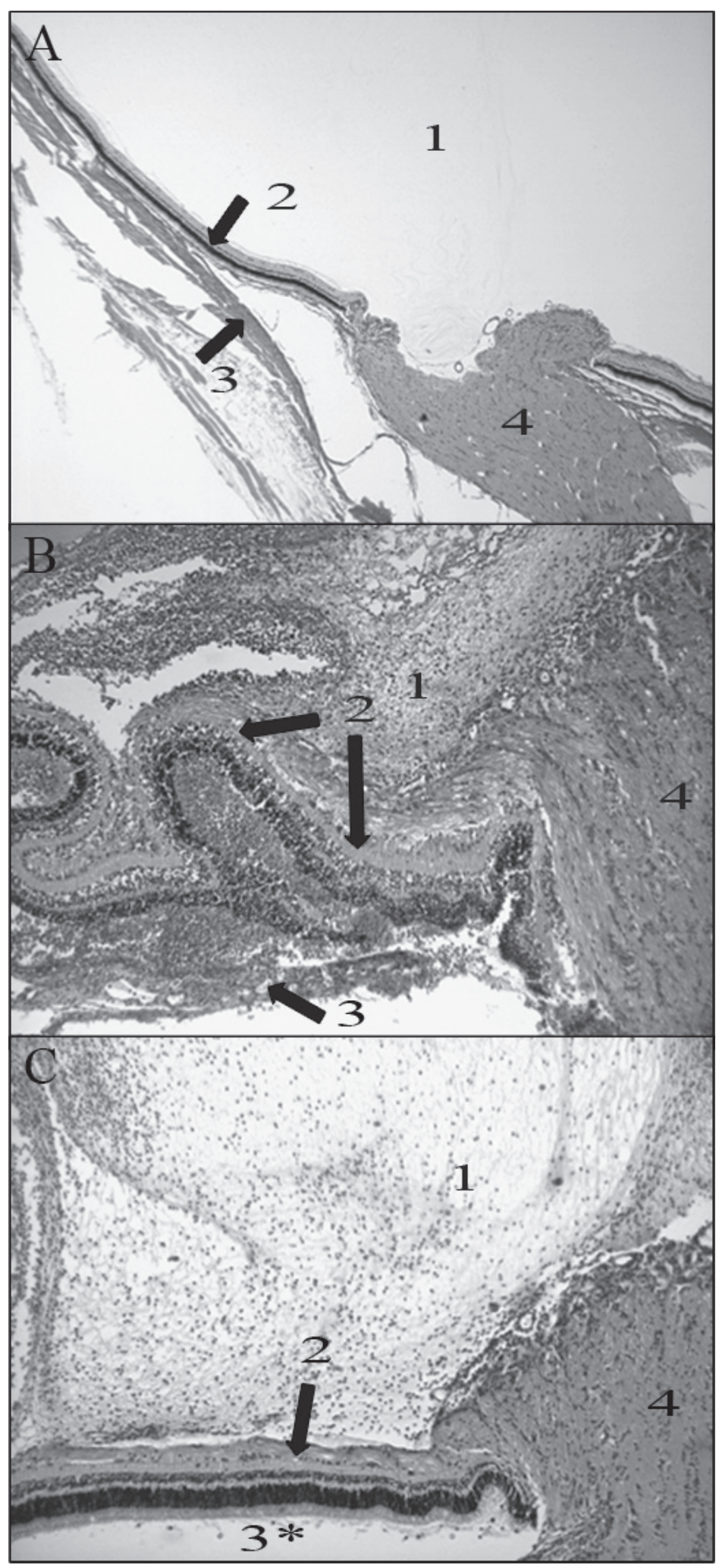

Figure 4 Representative histology pictures of an uninfected eye (A; 20X magnification) and eyes infected with strains producing high (B; 40X magnification) or low (C; 40X magnification) PLY activity. I is vitreous; 2 is retinal layers; 3 is choroid; 4 is optic disk. (A) Normal vitreous (I), retina (top arrow), and choroid (bottom arrow) were observed in an uninfected eye. (B) Damage and inflammation of the retina were observed (top two arrows). The choroid had become separated from the retinal layers by PMNs (bottom arrow). Severe inflammation was observed in the vitreous (I). (C) Only moderate inflammation was observed in the vitreous (I). Minor inflammation was observed in the retinal layers (arrow).

Notes: $3 *$ Subretinal space; choroid not visible in photograph due to artifactual separation from retina.

Abbreviations: PLY, pneumolysin; PMNs, polymorphonuclear cells. 
rat vitreous infected with $S$. pneumoniae strains deficient in the genes for PLY and autolysin, a pneumococcal protein that causes autolysis and daughter cell separation, had less severe endophthalmitis than the parent strain at 24 hours PI (Ng et al 2002). However, by 48 hours PI, there were no differences between the parent strain and the PLY-deficient and autolysin-deficient strains in the severity of endophthalmitis. These data prompted a suggestion that PLY and autolysin could contribute to early endophthalmitis, especially inflammation, and that other virulence factors may be involved in the disease ( $\mathrm{Ng}$ et al 2002). A clinical implication of the previous and current findings regarding the role of PLY in pneumococcal endophthalmitis would be the development of strategies to inhibit PLY, especially during the early stages of endophthalmitis.

The study presented herein used 5 clinical endophthalmitis strains as opposed to the D39 strain that was used in the previous study ( $\mathrm{Ng}$ et al 2002) and that was described by Avery (1944) and coworkers. Recent analysis of the genome sequences of different pneumococcal strains has indicated that pneumococcal genomes are diverse (Hoskins et al 2001; Tettelin et al 2001; Lanie et al 2007) and that different strain types cause different disease patterns (Blue and Mitchell 2003; Hava et al 2003; Orihuela et al 2003; Lanie et al 2007). This diversity between strains likely accounts for the differences in hemolytic activity, PLY concentration, and pathogenesis observed in the current study. This study also showed that PLY production is not a reliable indicator of hemolytic activity or the pathogenesis of $S$. pneumoniae endophthalmitis. Examination of the differences in hemolytic activity between a variety of strains, as well as the identification and characterization of other possible pneumococcal virulence factors in endophthalmitis, will aid in determining the mechanisms of this disease.

\section{Acknowledgments}

The authors would like to thank Dr. Hilary Thompson and Dr William Johnson for their consultation in using the appropriate statistics for this study. The authors report no conflicts of interest in this work.

\section{References}

Avery OT, MacLeod CM, McCarty M. 1944. Studies on the chemical nature of the substance inducing transformation of pneumococcal types: induction of transformation by a desoxyribonucleic acid fraction isolated from pneumococcus type III. J Exp Med, 79:137-58.

Beurg M, Hafidi A, Skinner L, et al. 2005. The mechanism of pneumolysininduced cochlear hair cell death in the rat. J Physiol, 568:211-27.

Blue CE, Mitchell TJ. 2003. Contribution of a response regulator to the virulence of Streptococcus pneumoniae is strain dependent. Infect Immun, 71:4925-35.
Callegan MC, Gilmore MS, Gregory M, et al. 2007. Bacterial endophthalmitis: therapeutic challenges and host-pathogen interactions. Prog Retin Eye Res, 26:189-203.

Callegan MC, Jett BD, Hancock LE, et al. 1999. Role of hemolysin BL in the pathogenesis of extraintestinal Bacillus cereus infection assessed in an endophthalmitis model. Infect Immun, 67:3357-66.

Canvin JR, Paton JC, Boulnois GJ, et al. 1997. Streptococcus pneumoniae produces a second haemolysin that is distinct from pneumolysin. Microb Pathog, 22:129-32.

Cima-Cabal MD, Mendez FJ, Vazquez F, et al. 2001. A specific and ultrasensitive chemiluminescent sandwich ELISA test for the detection and quantitation of pneumolysin. J Immunoassay Immunochem, 22:99-112.

Comis SD, Osborne MP, Stephen J, et al. 1993. Cytotoxic effects on hair cells of guinea pig cochlea produced by pneumolysin, the thiol activated toxin of Streptococcus pneumoniae. Acta Otolaryngol, 113:152-9.

Garcia-Suarez Mdel M, Florez N, Astudillo A, et al. 2007. The role of pneumolysin in mediating lung damage in a lethal pneumococcal pneumonia murine model. Respir Res, 8:3.

Green SN, Sanders M, Moore QC, et al. 2008. Protection from Streptococcus pneumoniae keratitis by passive immunization with pneumolysin antiserum. Invest Ophthalmol Vis Sci, 49:290-94.

Hava DL, Hemsley CJ, Camilli A. 2003. Transcriptional regulation in the Streptococcus pneumoniae rlrA pathogenicity islet by RlrA. J Bacteriol, 185:413-21.

Hirst RA, Gosai B, Rutman A, et al. 2008. Streptococcus pneumoniae deficient in pneumolysin or autolysin has reduced virulence in meningitis. J Infect Dis, 197:744-51.

Hoskins J, Alborn WE, Jr., Arnold J, et al. 2001. Genome of the bacterium Streptococcus pneumoniae strain R6. J Bacteriol, 183:5709-17.

Johnson MK, Callegan MC, Engel LS, et al. 1995. Growth and virulence of a complement-activation-negative mutant of Streptococcus pneumoniae in the rabbit cornea. Curr Eye Res, 14:281-4.

Johnson MK, Hobden JA, Hagenah M, et al. 1990. The role of pneumolysin in ocular infections with Streptococcus pneumoniae. Curr Eye Res, 9:1107-14.

Jounblat R, Kadioglu A, Mitchell TJ, et al. 2003. Pneumococcal behavior and host responses during bronchopneumonia are affected differently by the cytolytic and complement-activating activities of pneumolysin. Infect Immun, 71:1813-9.

Kirkham LS, Kerr AR, Douce GR, et al. 2006. Construction and immunological characterization of a novel nontoxic protective pneumolysin mutant for use in future pneumococcal vaccines. Infect Immun, 74:586-93.

Lanie JA, Ng W, Kazmierczak KM, et al. 2007. Genome sequence of Avery's virulent serotype 2 strain D39 of Streptococcus pneumoniae and comparison with that of unencapsulated laboratory strain R6. J Bacteriol, 189:38-51.

Mao LK, Flynn HW, Jr, Miller D, et al. 1992. Endophthalmitis caused by streptococcal species. Arch Ophthalmol, 110:798-801.

Marquart ME, Monds KS, McCormick CC, et al. 2007. Cholesterol as treatment for pneumococcal keratitis: cholesterol-specific inhibition of pneumolysin in the cornea. Invest Ophthalmol Vis Sci, 48:2661-6.

Mengaud J, Chenevert J, Geoffroy C, et al. 1987. Identification of the structural gene encoding the $\mathrm{SH}$-activated hemolysin of Listeria monocytogenes: listeriolysin $\mathrm{O}$ is homologous to streptolysin $\mathrm{O}$ and pneumolysin. Infect Immun, 55:3225-7.

Miller JJ, Scott IU, Flynn HW, et al. 2004. Endophthalmitis caused by Streptococcus pneumoniae. Am J Ophthalmol, 138:231-6.

$\mathrm{Ng}$ EW, Costa JR, Samiy N, et al. 2002. Contribution of pneumolysin and autolysin to the pathogenesis of experimental pneumococcal endophthalmitis. Retina, 22:622-32.

$\mathrm{Ng}$ EW, Samiy N, Cousins FV, et al. 1997. Implication of pneumolysin as a virulence factor in Streptococcus pneumoniae endophthalmitis. Retina, 17:521-9 
Nouri M, Terada H, Alfonso EC, et al. 2001. Endophthalmitis after keratoprosthesis: incidence, bacterial causes, and risk factors. Arch Ophthalmol, 119:484-9.

Orihuela CJ, Gao G, McGee M. 2003. Organ-specific models of Streptococcus pneumoniae disease. Scand J Infect Dis, 35:647-52.

Paton JC, Rowan-Kelly B, Ferrante A. 1984. Activation of human complement by the pneumococcal toxin pneumolysin. Infect Immun, 43:1085-7.

Quin LR, Moore QC, McDaniel LS. 2007. Pneumolysin, PspA, and PspC contribute to pneumococcal evasion of early innate immune responses during bacteremia in mice. Infect Immun, 75:2067-70.

Rubins JB, Charboneau D, Paton JC, et al. 1995. Dual function of pneumolysin in the early pathogenesis of murine pneumococcal pneumonia. J Clin Invest, 95:142-50.

Soriano F, Perez-Trallero E, Pallares R, et al. 2006. Streptococcus pneumoniae endophthalmitis: a study of 36 cases with special reference to antibiotic resistance and treatment options. Clin Microbiol Infect, 12:519-26.
Tettelin H, Nelson KE, Paulsen IT, et al. 2001. Complete genome sequence of a virulent isolate of Streptococcus pneumoniae. Science, 293:498.

Thornton J, McDaniel LS. 2005. THP-1 monocytes up-regulate intercellular adhesion molecule 1 in response to pneumolysin from Streptococcus pneumoniae. Infect Immun, 73:6493-8.

Witzenrath M, Gutbier B, Hocke AC, et al. 2006. Role of pneumolysin for the development of acute lung injury in pneumococcal pneumonia. Crit Care Med, 34:1947-54.

Yuste J, Botto M, Paton JC, et al. 2005. Additive inhibition of complement deposition by pneumolysin and PspA facilitates Streptococcus pneumoniae septicemia. J Immunol, 175:1813-9. 